\title{
Advantages of the quenching probe method over other PCR-based methods for detection of the $J A K 2$ V617F mutation
}

\author{
AYA ONO, YUKI OKUHASHI, YUSUKE TAKAHASHI, MAI ITOH, NOBUO NARA and SHUJI TOHDA \\ Department of Laboratory Medicine, Tokyo Medical and Dental University, Tokyo 113-8519, Japan
}

Received February 13, 2012; Accepted May 29, 2012

DOI: $10.3892 / \mathrm{ol} .2012 .741$

\begin{abstract}
The detection of a V617F mutation (G to T exchange at nucleotide 1,849) in the $J A K 2$ gene is crucial for the diagnosis of myeloproliferative neoplasms (MPN) such as polycythemia vera. Although sequence analysis is the standard method for detection, it is not suitable for clinical examinations due to the requirement of expensive equipment. In this study, we evaluated the efficiencies of four PCR-based methods to detect JAK2 V617F: allele-specific PCR (AS-PCR), PCR-restriction fragment length polymorphism (PCR-RFLP), high-resolution melting analysis (HRM) and the quenching probe method (QP). The HEL cell line, which harbors a homozygous JAK2 $\mathrm{V} 617 \mathrm{~F}$ mutation, as well as bone marrow samples from 16 MPN patients and normal control samples, were used in this assessment. The sensitivity of the detection limit of all four methods was also examined using samples of HEL cells mixed in a variety of ratios with cells containing wild-type $J A K 2$. The results of all four methods were found to be concordant. AS-PCR was shown to be the most sensitive; however, it produced false positive results. Although PCR-RFLP demonstrated high specificity, it was time consuming. By contrast, results were obtained using HRM and QP in only $2 \mathrm{~h}$. It was easier to recognize the curves derived from the mutant allele obtained using QP. QP is also suitable for the rough estimation of allele burden. JAK2 V617F assays are mainly used for diagnosis at presentation in clinical settings. We therefore conclude that in situations where high sensitivity is not required, QP is the preferable method for the detection of JAK2 V617F. To the best of our knowledge, this is the first report to demonstrate the efficiency of the QP method for the detection of JAK2 V617F using a standard thermal cycler.
\end{abstract}

Correspondence to: Dr Shuji Tohda, Department of Laboratory Medicine, Tokyo Medical and Dental University, Yushima 1-5-45, Bunkyo-Ku, Tokyo 113-8519, Japan

E-mail: tohda.mlab@tmd.ac.jp

Key words: janus kinase 2, myeloproliferative neoplasms, allele-specific polymerase chain reaction, PCR-restriction fragment length polymorphism, high-resolution melting analysis, quenching probe

\section{Introduction}

Janus kinase 2 (JAK2) is a cytoplasmic tyrosine kinase that has roles in hematopoietic growth factor signaling pathways such as those stimulated by erythropoietin and interleukin-3. An acquired mutation of $\mathrm{G}$ to $\mathrm{T}$ at nucleotide 1,849 of JAK2, JAK2 $\mathrm{V} 617 \mathrm{~F}$, constitutively activates signaling in hematopoietic stem cells and leads to the development of myeloproliferative neoplasms (MPN) such as polycythemia vera (PV), essential thrombocythemia (ET), and primary myelofibrosis (PMF). Indeed, JAK2 mutation is included in the diagnostic criteria of these three diseases (1).

Although sequence analysis is currently the standard method for the detection of JAK2 mutation, this technique is not suitable for clinical examinations in hospital laboratories as it requires expensive equipment and takes time. Moreover, the sensitivity of the detection limit of sequencing is not less than $10-20 \%$ (2). Therefore, the use of various PCR-based methods such as allele-specific PCR (AS-PCR), PCR-restriction fragment length polymorphism (PCR-RFLP) and high-resolution melting analysis (HRM) has been investigated. Recently, the quenching probe method (QP) has been attracting attention as a novel technique capable of detecting single nucleotide polymorphisms and mutations (3).

In QP, an oligonucleotide with a cytosine modified by fluorescent dye at the $3^{\prime}$ end (Q probe) is used as the probe. Following completion of PCR, a melting curve analysis is performed. At low temperature, the probes hybridize with PCR products and their fluorescence is quenched by an electron transfer to adjacent guanine bases in the PCR products. As the temperature is raised, the probes dissociate from the PCR products and the fluorescent signal increases. Since the probes dissociate from unmatched products at lower temperatures than perfectly matched products, it is possible to detect mutations.

In this study, we performed a comparative evaluation of the efficiency and sensitivity of four PCR-based methods to detect JAK2 V617F. To the best of our knowledge, this is the first study to demonstrate the efficiency of the QP method for the detection of $J A K 2 \mathrm{~V} 617 \mathrm{~F}$ using a standard thermal cycler.

\section{Materials and methods}

Cells and DNA extraction. Two leukemia cell lines were used. HEL, an erythroid leukemia cell line with a homozygous JAK2 
Table I. Primers, probes and PCR protocols.

\begin{tabular}{|c|c|c|c|c|}
\hline Method & Primer sequence & No. of cycles & Melting point temperature & Ref. \\
\hline AS-PCR & $\begin{array}{l}\text { For: 5'-atctatagtcatgctgaaagtaggagaaag-3' } \\
\text { Mut: 5'-agcatttggttttaaattatggagtatatt--3' } \\
\text { Rev: 5'-ctgaatagtcctacagtgtttcagtttca-3' }\end{array}$ & 40 & $94^{\circ} \mathrm{C} 1 \mathrm{~min}, 57^{\circ} \mathrm{C} 1 \mathrm{~min}, 72^{\circ} \mathrm{C} 1 \mathrm{~min}$ & 4 \\
\hline \multicolumn{5}{|l|}{ PCR-RFLP } \\
\hline First round & $\begin{array}{l}\text { For: 5'-ggtttcctcagaacgttgatgg-3' } \\
\text { Rev: } 5 \text { '-ttgtttgggcattgtaaccttc-3' }\end{array}$ & 35 & $94^{\circ} \mathrm{C} 1 \mathrm{~min}, 55^{\circ} \mathrm{C} 1 \min , 72^{\circ} \mathrm{C} 1 \min$ & 5 \\
\hline Second round & $\begin{array}{l}\text { For: 5'-tgctgaaagtaggagaaagtgcat-3' } \\
\text { Rev: 5'-tcctacagtgttttcagtttcaaaaa-3' }\end{array}$ & 25 & $94^{\circ} \mathrm{C} 1 \mathrm{~min}, 60^{\circ} \mathrm{C} 1 \mathrm{~min}, 72^{\circ} \mathrm{C} 1 \mathrm{~min}$ & \\
\hline HRM & $\begin{array}{l}\text { For: 5'-agcaagctttctcacaagca-3' } \\
\text { Rev: 5'-ctgacacctagctgtgatcctg-3' }\end{array}$ & 45 & $95^{\circ} \mathrm{C} 15 \mathrm{sec}, 58^{\circ} \mathrm{C} 15 \mathrm{sec}, 72^{\circ} \mathrm{C} 15 \mathrm{sec}$ & 6 \\
\hline $\mathrm{QP}$ & $\begin{array}{l}\text { For: 5'-gcagcaagtatgatgagcaagctttctc-3' } \\
\text { Rev: 5'-gctctgagaaaggcattagaaagcctg-3' } \\
\text { Probe: 5'-agtatgtttctgtggagac-(BODIPY FL)-3' }\end{array}$ & 45 & $95^{\circ} \mathrm{C} 15 \mathrm{sec}, 58^{\circ} \mathrm{C} 15 \mathrm{sec}, 72^{\circ} \mathrm{C} 15 \mathrm{sec}$ & 7 \\
\hline
\end{tabular}

The primer and probe sequences correspond to those in the indicated references. The base corresponding to the mutated nucleotide 1,849 is underlined.

V617F mutation, was supplied by the Japanese Collection of Research Bioresources (Ibaraki, Japan). KOPT-K1, a T-lymphoblastic leukemia cell line with wild-type $J A K 2$, was donated by Drs. Harashima and Orita (Fujisaki Cell Center, Japan). To investigate the sensitivity of the detection limit, samples of HEL cells and KOPT-K1 cells mixed at various ratios were used. We obtained bone marrow samples from 16 MPN patients (six PV, eight ET, one PMF, and one unclassifiable MPN) with informed consent. Blood samples from two normal volunteers were used as a control. DNA was extracted from cells following the spin column method using QIAamp DNA Blood Mini kit (Qiagen, Germantown, MD, USA).

AS-PCR. The schematic outlines of all four PCR methods are shown in Fig. 1, while the primer sequences used are presented in Table I. The AS-PCR method used in this study was modified from a previously reported protocol (4) and the mutant primer contained an intentional mismatch at the third nucleotide from the 3 ' end to improve specificity. Amplifications were performed in a $50 \mu 1$ reaction volume with $0.2 \mu \mathrm{g}$ DNA, $1.5 \mathrm{mM} \mathrm{MgCl}_{2}, 0.2 \mathrm{mM}$ dNTP, $0.5 \mu \mathrm{M}$ primers, and 1 unit Taq polymerase (Takara, Japan), and the resulting PCR products were electrophoresed in a $2 \%$ agarose gel and stained with ethidium bromide. The assay was repeated at least three times to ascertain the reproducibility of this method.

PCR-RFLP. The PCR-RFLP method used in this study was modified from a previously reported protocol (5). The first round of nested PCR was performed in a $50 \mu \mathrm{l}$ reaction volume with $0.2 \mu \mathrm{g}$ DNA sample, $1.5 \mathrm{mM} \mathrm{MgCl}_{2}, 0.2 \mathrm{mM}$ dNTP, $0.5 \mu \mathrm{M}$ primers, and 1 unit Taq polymerase. The second round of PCR was performed in a $50 \mu$ l reaction volume containing $2 \mu 1$ of the first round products. The resulting products were digested with BsaXI (New England Biolabs, Hitchin, UK), and the PCR products and BsaXI-treated products were electrophoresed as described above.

HRM. HRM was performed using a LightCycler Nano (Roche Diagnostics, Mannheim, Germany) according to a previously reported protocol, with modifications (6). Amplifications were performed in a $20 \mu 1$ reaction volume with 30 ng DNA sample, $3 \mathrm{mM} \mathrm{MgCl} 2,0.2 \mu \mathrm{M}$ primers and $10 \mu 1$ Master Mix [LC 480 High Resolution Melting Master (Roche)] following incubation for $10 \mathrm{~min}$ at $95^{\circ} \mathrm{C}$. Melting conditions were as follows: denaturation at $95^{\circ} \mathrm{C}$ for $1 \mathrm{~min}$, renaturation at $40^{\circ} \mathrm{C}$ for $1 \mathrm{~min}$, and melting at a gradient from $60-95^{\circ} \mathrm{C}$ with 50 fluorescent acquisitions per $1^{\circ} \mathrm{C}$. The normalized and temperature-shifted difference curves were determined using LightCycler Nano HRM software (Roche).

$Q P$. The QP method was performed with modifications to a previously reported protocol (7) using a LightCycler Nano. Following incubation for $10 \mathrm{~min}$ at $95^{\circ} \mathrm{C}$, amplifications were performed in a $20 \mu \mathrm{l}$ reaction volume with $30 \mathrm{ng}$ DNA sample, $1.5 \mathrm{mM} \mathrm{MgCl}_{2}, 0.2 \mu \mathrm{M}$ primers, $0.1 \mu \mathrm{M}$ Q probe, and $10 \mu \mathrm{l}$ reaction mix [LC 480 Genotyping Master (Roche)]. The Q probe was labeled with BODIPY FL at its 3' end, and had a sequence complementary to the mutant allele. Following completion of PCR, the temperature was maintained at $95^{\circ} \mathrm{C}$ for $1 \mathrm{~min}, 40^{\circ} \mathrm{C}$ for $1 \mathrm{~min}$, and then gradually increased to $90^{\circ} \mathrm{C}$ at the rate of $0.05^{\circ} \mathrm{C} / \mathrm{sec}$, during which the fluorescence signal was continually acquired. The curves for degree of fluorescence increase $(\mathrm{dF} / \mathrm{dT})$ were obtained from the data using LightCycler Nano software (Roche).

Direct sequencing. To confirm the $\mathrm{G}$ to $\mathrm{T}$ mutation at nucleotide 1,849 , the PCR products from selected samples were sequenced using a 3130xl Genetic Analyzer and BigDye v3.1 (Applied Biosystems, Foster City, CA, USA). 


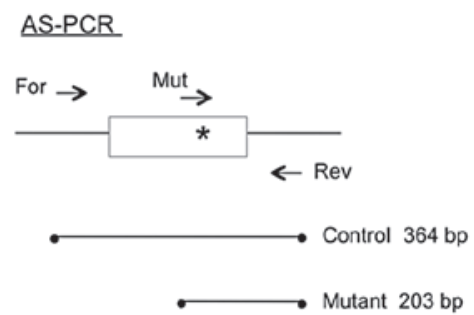

$\underline{\text { HRM }}$

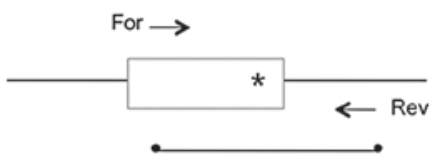

PCR-RFLP

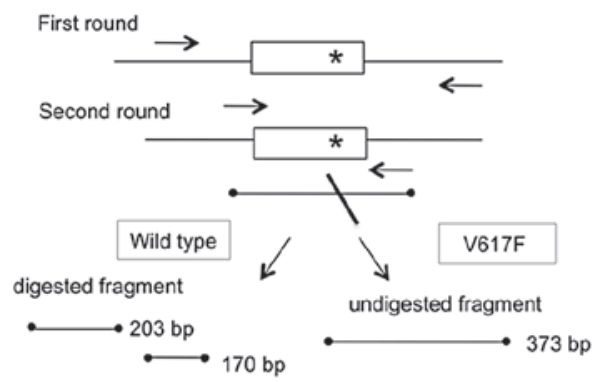

$\underline{Q P}$

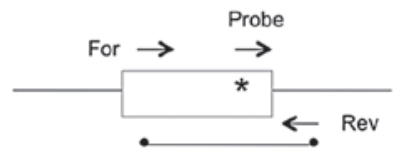

Figure 1. Schematic outline of four PCR-based detection methods for JAK2 V617F mutation.

\section{Results}

Discrimination between wild-type and mutant alleles. The AS-PCR method produced a 364-bp band from wild-type and mutant alleles, as well as a 203-bp band from the mutant allele (upper panel of Fig. 2). The nested PCR products obtained by PCR-RFLP were 373 bp in size. Following BsaXI digestion, the products from the wild-type allele were cut into 203-bp and 170-bp fragments while the products from the mutant allele remained unchanged (lower panel of Fig. 2). With the HRM method, the melting profile of the wild-type control was designated as the horizontal line to which the normalized and temperature-shifted difference curves were drawn. The samples containing mutant products are distinguishable by their concave-up curves (left panel of Fig. 3). Finally, with the QP method, the wild-type allele produced concave-up curves with the lowest point at $51^{\circ} \mathrm{C}$, while the mutant allele produced concave-up curves with the lowest point at $60^{\circ} \mathrm{C}$ (right panel of Fig. 3).

Detection of the JAK2 V617F mutation in patient samples. Of the 16 patient samples, 7 (3 PV, $3 \mathrm{ET}$, zero PMF, and one unclassifiable MPN) were determined to be V617F-positive. Although the results obtained by AS-PCR from a few samples were initially discrepant with those obtained via the other methods, this was settled by adjusting the $\mathrm{MgCl}_{2}$ concentration and annealing temperature. The results from the four PCR-based methods and direct sequencing were ultimately found to be concordant.

Sensitivity of detection. We examined the sensitivity of the detection limit using DNA extracted from samples containing mixtures of HEL and KOPT-K1 cells in various ratios. Fig. 2 shows representative results from AS-PCR and PCR-RFLP. The numbers indicate the percentage of HEL cells in the given sample. Although the mutant bands obtained by AS-PCR from low percentage samples are not clearly visible in this photograph, the bands from the 0.2-0.5\% mutant samples were visible on a transilluminator. The PCR-RFLP method detected

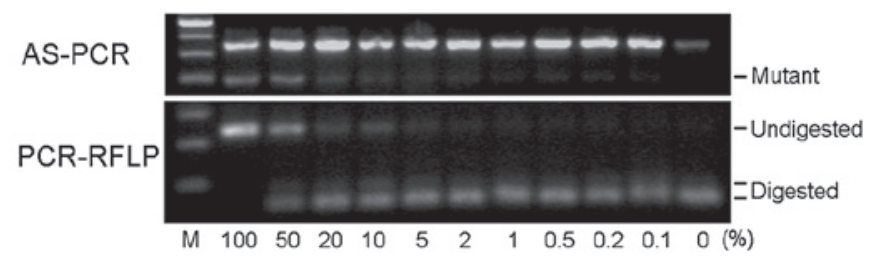

Figure 2. Sensitivity of detection limit of AS-PCR and PCR-RFLP for JAK2 V617F mutation. DNA extracted from samples containing mixtures of HEL and KOPT-K1 cells was used; numbers indicate the percentage of HEL cells in the given sample. The PCR products (upper panel) and the nested PCR products digested with BsaXI (lower panel) were electrophoresed in an agarose gel. $\mathrm{M}, 100 \mathrm{bp}$ marker.
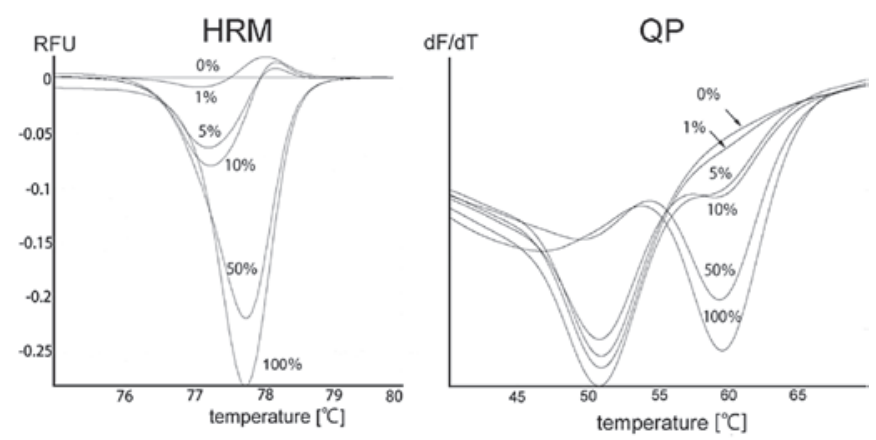

Figure 3. Sensitivity of HRM and QP for the detection of JAK2 V617F mutation. DNA extracted from samples containing mixtures of HEL and KOPT-K1 cells was used; numbers indicate the percentage of HEL cells in the given sample.

mutants in 1-2\% samples. As shown in the left panel of Fig. 3, the samples containing 1-5\% mutant allele were distinguishable by their concave-up curves on HRM. Finally, QP detected the mutant allele in samples containing 5\% mutant, as evidenced by the concave-up curves with the lowest point at $60^{\circ} \mathrm{C}$ of the samples (right panel of Fig. 3). 
Table II. Comparison of PCR methods.

\begin{tabular}{lccll}
\hline Method & Sensitivity $(\%)$ & Time $(\mathrm{h})$ & \multicolumn{1}{c}{ Strengths } & \multicolumn{1}{c}{ Weaknesses } \\
\hline AS-PCR & $0.2-0.5$ & 4.5 & High sensitivity & Possible false positive \\
PCR-RFLP & $1-2$ & 9.5 & High specificity & Time consuming \\
HRM & $1-5$ & 2.0 & Fast results & $\begin{array}{l}\text { Difficult to identify the mutant } \\
\text { curve at low percentages } \\
\text { Requires high quality DNA }\end{array}$ \\
QP & 5 & 2.0 & $\begin{array}{l}\text { Easy to recognize the } \\
\text { mutant curve }\end{array}$ & \\
\hline
\end{tabular}

Sensitivity refers to the detection limit obtained in experiments using samples of mixed HEL and KOPT-K1 cells. Time is the number of hours required for results to be obtained after DNA extraction.

\section{Discussion}

In this study, we compared the efficiency of four PCR-based methods to detect JAK2 V617F (Table II). AS-PCR was found to be the most sensitive method of the four, although this method did have weaknesses. Firstly, this method was initially found to produce false-positive results in a small number of samples due to the annealing of the mutant primer to the wild-type allele. Secondly, it is not possible to estimate the mutant allele burden using this method. To cope with this weakness, another AS-PCR was carried out according to a previously reported protocol using two primers for the mutant allele and wild-type allele individually, in addition to the outer forward and reverse primers (8). However, the sensitivity and reproducibility were not as good with this protocol, likely due to competition of the primers (data not shown).

The PCR-RFLP method did not result in false-positive and false-negative results in this study. Furthermore, this method was able to approximately estimate the mutant allele burden by comparing the density of the digested bands and undigested band. However, the weakness of this method is that it is time consuming, taking $9.5 \mathrm{~h}$ to obtain results. While it was possible to obtain results after the first round of PCR and subsequent enzyme digestion from cell line samples, detection in patient samples required nested PCR.

By contrast, HRM and QP are able to produce results in $2 \mathrm{~h}$ as there is no need for gel electrophoresis with these methods. However, high purity DNA samples are required; crude DNA samples did not produce clear results due to non-specific PCR products that caused the vacillation of the curves. Therefore, a spin-column method should be used for DNA extraction rather than a phenol/chloroform or agglutination partition method. When HRM and QP were compared, it was easier to recognize the curves derived from the mutant alleles using the QP method as the curves created by the wild-type allele and mutant allele have different positions on the temperature axis. Moreover, it is possible to roughly estimate allele burden quantification following QP by comparing the sizes of the curves at $51^{\circ} \mathrm{C}$ to those at $60^{\circ} \mathrm{C}$, as well as the proportions of two curves from a given patient sample to that shown in Fig. 3 .

Taken together, AS-PCR was found to be the best method for the detection of JAK2 V617F in terms of sensitivity, while QP was the best method in terms of promptness and ease of interpretation. In clinical settings, JAK2 V617F assays are mainly used for diagnosis at presentation rather than for detecting minimal residual diseases. As cells expressing the mutant allele usually account for more than $10 \%$ of the samples in these cases, high sensitivity is not necessarily required. We therefore suggest that QP would be the preferable method for $J A K 2$ V617F detection in clinical examination in hospitals.

\section{Acknowledgements}

We thank Dr N. Murakami and Dr T. Fukuda (Tokyo Medical and Dental University) for their assistance in obtaining samples from the patients. This study was supported in part by a Grant-in-Aid for Scientific Research (C) from the Japan Society for the Promotion of Science (No. 18690522).

\section{References}

1. Thiele J, Kvasnycka HM, Orazi A, Tefferi A and Birgegard G: Polycythemia vera. In: WHO Classification of Tumours of Haematopoietic and Lymphoid Tissues. Swerdlow SH, Campo E, Harris NL, Jaffe ES, Pileri ES, Stein H, Thiele J and Vardiman JW (eds). IARC Press, Geneva, pp40-43, 2008.

2. James C, Ugo V, Le Couédic JP, et al: A unique clonal JAK2 mutation leading to constitutive signalling causes polycythaemia vera. Nature 434: 1144-1148, 2005.

3. Crockett AO and Wittwer CT: Fluorescein-labeled oligonucleotides for real-time PCR: using the inherent quenching of deoxyguanosine nucleotides. Anal Biochem 290: 89-97, 2001.

4. Baxter EJ, Scott LM, Campbell PJ, et al: Acquired mutation of the tyrosine kinase JAK2 in human myeloproliferative disorders. Lancet 365: 1054-1061, 2005.

5. Horn T, Kremer M, Dechow T, et al: Detection of the activating JAK2 V617F mutation in paraffin-embedded trephine bone marrow biopsies of patients with chronic myeloproliferative diseases. J Mol Diagn 8: 299-304, 2006.

6. Er TK, Lin SF, Chang JG, et al: Detection of the JAK2 V617F missense mutation by high resolution melting analysis and its validation. Clin Chim Acta 408: 39-44, 2009.

7. Tanaka R, Kuroda J, Stevenson W, et al: Fully automated and super-rapid system for the detection of JAK2V617F mutation. Leuk Res 32: 1462-1467, 2008.

8. Jones AV, Kreil S, Zoi K, et al: Widespread occurrence of the JAK2 V617F mutation in chronic myeloproliferative disorders. Blood 106: 2162-2168, 2005. 